\title{
Maximization of Fundamental Frequency of Laminated Composite Cylindrical Shells by Ant Colony Algorithm
}

\author{
Rubem Matimoto Koide, Marco Antonio Luersen
}

\begin{abstract}
The success in developing modern aerospace systems addresses competitive subjects as optimization, reduced costs, sustainability, environment, weight, and safety. There is an increased demand for lighter materials such as laminated composites. In order to match the demand of aeronautical companies, the shell structures are very important. The dynamic behavior of composite structures is also essential to improve the potential applications of these materials. The knowledge of the dynamic response of the cylindrical shell structures is an important issue in their design, in which the ply thicknesses are often preestablished and the ply orientations are usually restricted to a small set of angles due to manufacturing constraints. Obtaining the best stacking sequence of laminated shell may lead to a problem of combinatorial optimization. As this problem is hard to be solved, several techniques have been developed. Ant colony optimization is a class of heuristic optimization algorithms inspired by the behavior of real ants, related to their ability to find the shortest path between the nest and the food source. Thus, this paper deals with optimal stacking sequence of laminated cylindrical composite shells with the aim of maximizing their fundamental frequency using this approach. The ant colony algorithm was implemented in Matlab platform and was linked to Abaqus academic version to compute the structural response. Cylindrical shells with and without a cutout geometry were studied. Fundamental frequencies were maximized for both cases, and results were presented and discussed.
\end{abstract}

KEYWORDS: Frequency maximization, Laminated cylindrical shells, Ant colony optimization, Heuristic optimization.

\section{INTRODUCTION}

Many successful applications of composite materials and the development of modern aerospace systems are to address competitive subjects, such as optimization, reduced costs, sustainability, environment, weight, and safety. The demand of aeronautical companies for lighter, safer, and less polluting airliners is increasing for the next generations of aircraft, as related by Irisarri et al. (2011), mainly to integrate primary structures such as the centre wing box, fuselage, and wings. As reinforced by Todoroki et al. (2011), for laminated composite structures, the stacking sequence optimization is indispensable. These structures are widely used for aerospace components due to their high specific strength and specific stiffness. Increase use of laminated composite shells has motivated research in shell dynamic behavior. Thus, Shafei and Kabir (2011) analyzed the optimization techniques for improvement of structural elements designs regarding vibration stability or modal frequencies. The dynamic behavior study about composite structures is essential for assessing their full potential, as concluded by Sharma and Mittal (2010). The analysis of natural frequencies of composite/shells plays an increasingly important role in the design of aerospace engineering applications (Sharma and Mittal, 2010). In this way, an optimum structural design aimed at improving stable vibration frequency or the dynamic response was created.

This paper has dealt with optimal stacking sequence design of laminated cylindrical composite shells. The stacking

1. Universidade Tecnológica Federal do Paraná - Curitiba/PR - Brazil

三 $\quad$ Author for correspondence: Rubem Matimoto Koide | Laboratório de Mecânica Estrutural (LaMEs]/Universidade Tecnológica Federal do Paraná | Avenida Sete de 
sequence optimization is formulated as a combinatorial problem and is solved using ant colony optimization (ACO), which is a class of heuristic algorithms proposed to solve optimization problems. The idea was inspired by the behavior of real ants, related to their ability to find the shortest path between the nest and the food source. Ant colony heuristic has been applied successfully to different kinds of problems. Due to their capacity to solve hard problem as the stacking sequence of laminated composite, the ACO was simulated in this study. The developed ant colony algorithm was applied to problems of maximizing the fundamental frequency of cylindrical shells with and without a cutout. ACO algorithm was coupled with a finite element code in order to obtain the structural response. Optimized stacking sequence results were presented and the modal frequency of cylindrical shell composite was also computed. The present work may help designers to optimize the dynamic response of complex structures without analytical solutions, and the optimization method may be applied in many others composite parts.

\section{ANT COLONY OPTIMIZATION APPLIED TO CYLINDRICAL SHELLS}

\section{ANT COLONY OPTIMIZATION}

ACO is a metaheuristic in which a colony of artificial ants cooperates in finding good solutions. This technique is applied to difficult discrete optimization problems. As described by Dorigo and Stützle (2004), the main procedure of the ACO metaheuristic manages scheduling of the three components of ACO algorithms via the Schedule Activities construct: management of the ant activity, pheromone updating, and daemon actions.

The pseudocode of ACO metaheuristic is presented by Dorigo and Stützle (2004) as;

\section{Procedure ACOmetaheuristic}

ScheduleActivities

ConstructAntsSolutions

UpdatePheromones

DaemonActions \% optional

End-ScheduleActivities

End-procedure
The ConstructionAntsSolutions is a procedure in which the solution of the optimization problem is built. Therefore, the artificial ants apply a stochastic decision rule in a problem defined by a construction graph. While the solution is being built, based on the pheromone trails and heuristic information, the ants evaluate the solutions for searching the optimal feasible candidate.

The Update Pheromone procedure is based on the solution available and it influences the quantity of pheromone. It can be increased or decreased. The deposit of new amount of pheromone increases the probability of a good solution in the next decision. The decrease process is related to pheromone evaporation. It influences in a choice of candidate due to the reduction of pheromone trail for the solution available, so a bad candidate is not selected. This process actives the convergence of the algorithm.

The DaemonActions procedure can be used as an optional process to optimize the algorithm. Local or global information can be used to decide or influence a search optimization procedure.

The Ant Colony System (ACS) is one extension of the first ant algorithm. Additional mechanisms in the ACS are: it exploits the search experience accumulated by the ants through the use of an aggressive action choice rule, as explained by Dorigo and Stützle (2004); the pheromone evaporation and deposit are updated only in a best-so-far connection.

ACS algorithm has a framework based on three rules that manage the optimization problem. In this variant, the first procedure is ConstructionAntsSolution or the pseudorandom proportional rule defined by Eq. 1:

$j=\left\{\begin{array}{l}\arg \max _{l \in N_{\mathrm{i}}^{\mathrm{K}}}\left\{\tau_{i l}\left[\eta_{i l}\right]^{\beta}\right\}, q \leq q_{0} \\ p_{i j}^{\mathrm{K}}=\frac{\left[\tau_{i l}\right]^{\alpha}\left[\eta_{i l}\right]^{\beta}}{\sum_{l \in N_{i}^{K}}\left[\tau_{i l}\right]^{\alpha}\left[\eta_{i l}\right]^{\beta}}, q \leq q_{0}\end{array}\right.$

where,

$q$ is a random variable uniformly distributed in $[0,1]$,

$q_{0}$ is a parameter for the best possible move $\left(0 \leq q_{0} \leq 1\right)$,

$k$ is an ant,

$\alpha$ is a parameter that determines the relative influence of the pheromone trail,

$\beta$ is a parameter that determines the relative influence of the heuristic information,

$\eta$ is the heuristic information value,

$i, j$ are the initial and next choice or candidate, 
$l$ is a candidate solution,

$N_{i}^{K}$ is the feasible neighborhood of ant $k$,

$p_{i j}^{K}$ is the probability that ant $k$ chooses the next solution if $q>q_{0}$.

If $q \leq q_{0}$, it means that the ant is exploiting the learned knowledge based on the pheromone trails and the heuristic information. If $q>q_{0}$, the ant explores other tours or searches around the best-so-far solution.

The second rule is the Global Pheromone Trail Update. In Eq. 2, the formulation for this update is:

$$
\tau_{i j} \leftarrow(1-\rho) \tau_{i j}+\rho \Delta \tau_{i j,}^{b s} \forall(i, j) \in T^{b s}
$$

where,

$\rho$ is the global pheromone evaporation rate $(0<\rho \leq 1)$,

$\Delta \tau_{i j}^{b s}$ is the amount of pheromone that ant $k$ deposits on each best-so-far solution,

$T^{b s}$ is a set of best connections.

The amount of pheromone is defined due to the objective function, in this case the inverse of the best-so-far fundamental frequency. When this rule is applied, both the evaporation and the new pheromone deposits are updated only to the best-so-far ant.

Local Pheromone Trail Update, the last rule, is applied during the tour construction. The pheromone evaporation and a new pheromone deposit are updated when an ant is exploiting or exploring the connection, according to the pseudorandom proportional rule. It is formulated by Eq. 3:

$$
\tau_{i j} \leftarrow(1-\xi) \tau_{i j}+\xi \tau_{0}
$$

where,

$\xi$ is the local pheromone evaporation rate $0<\xi<1$, and $\tau_{0}$ is the initial pheromone trails value.

The effect of the local updating rule is that each time an ant uses an $\operatorname{arc}(i, j)$ or connection, its pheromone trail $\tau_{i j}$ is reduced, so that the arc becomes less desirable for the following ants as explained by Dorigo and Stützle (2004).

\section{LAMINATED CYLINDRICAL SHELLS}

The laminated composite material consists of stacks of layers, each one usually composed by a matrix of polymeric material and fibers oriented in a specific direction. Laminated composites provide the designer the ability to tailor the material according to the application. The structures formed by these materials present high stiffness/ mass and strength/mass ratios. The mechanical properties of composite laminates depend on the material of each layer, the number of layers, the thickness of each layer, and the fiber orientations in each layer. The ply thicknesses are often predetermined and the ply orientations are usually restricted to a small set of angles due to manufacturing constraints. This fact leads to problems of discrete or stacking-sequence optimization. Figure 1 shows the laminated composite, the stacking sequence, and an application in cylindrical shell.

Composite cylindrical shell structures find wide applications in many products. These kinds of structures are in general efficient in engineering applications including aircraft, offshore platforms, silos, spacecraft, pressure vessels, pipelines, and many others industry applications. Considering these applications, many studies have been developed in this area. The general two-dimensional theory of laminated cylindrical shells was presented by Barbero et al. (1990). Heyliger and Jilani (1993) studied free vibration of laminated anisotropic cylindrical shells and presented numerical results for isotropic, anisotropic, and orthotropic composite shells. Soldatos (1994) approached a review of three dimensional dynamic analyses of circular cylinders and cylindrical shells. The analytical solutions were proposed by Lam and Qian (2000) for the free vibrations of symmetric angle-ply thick laminated composite cylindrical shell. Regarding noncircular cylindrical shells, Ganapathi and Haboussi (2003) have been developing free vibrations of thick laminates. Ganapathi et al. (2004) studied the free flexural vibration behavior of elliptical cylindrical shells.

The effects of shell geometry, cross-sectional properties, layup and ply-angle on the natural frequency, modes of vibrations, were evaluated for noncircular shell structures. Poore et al. (2008) also presented a free vibration analysis of laminated cylindrical shells with a circular cutout. Although advances in vibration studies involved complex mathematics analysis, experimental tests and recently numerical optimization methods, these subject studies are still under development. 


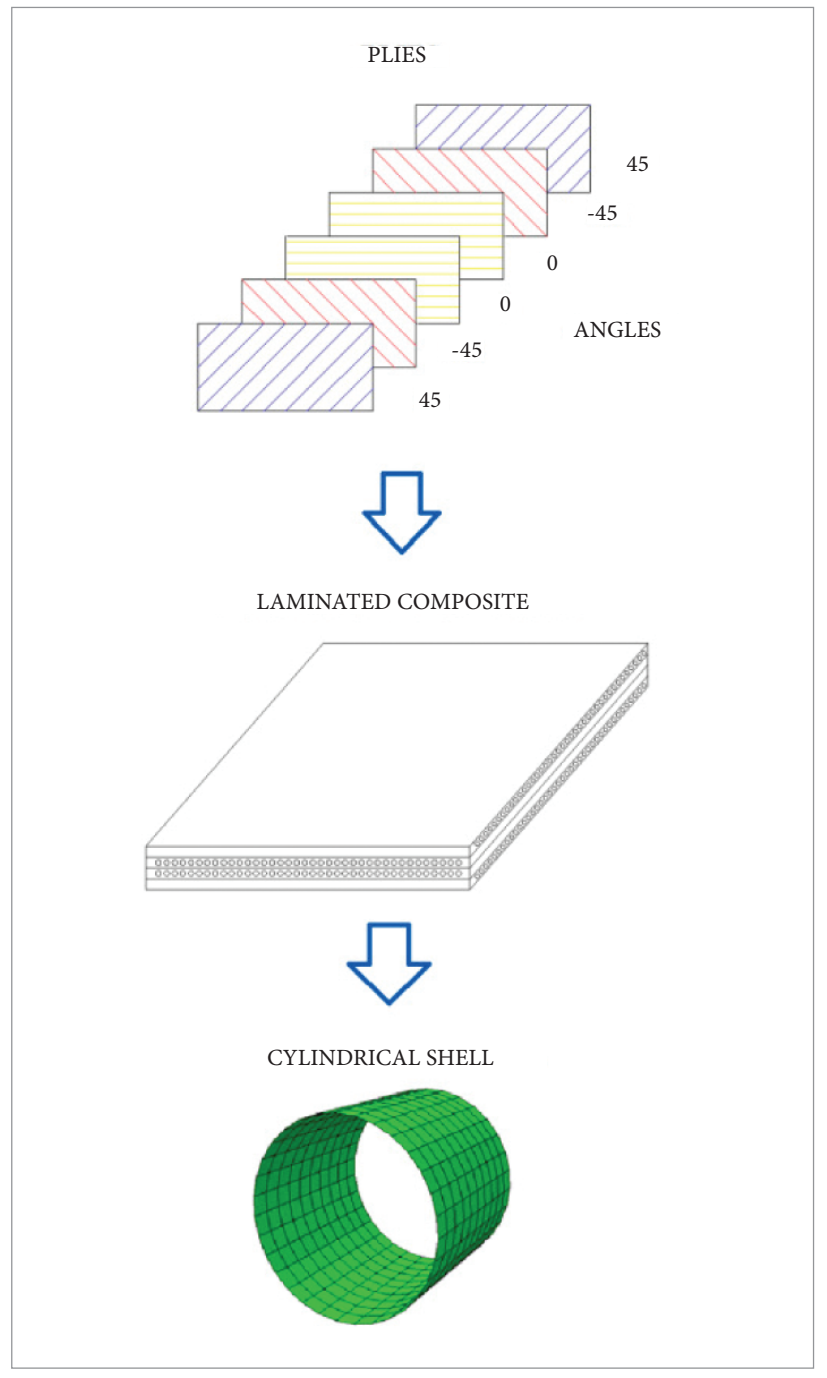

Figure 1. Laminated composite.

Since this optimization problem is very hard to solve, several techniques have been developed. Genetic algorithms (GA) have been successfully applied to solve these kinds of problems (Le Riche and Haftka, 1993).

Recently, a new class of algorithms, the ACO, was developed to solve combinatorial optimization problems. It was inspired by observing the behavior of real ants (Dorigo and Stützle, 2004). Regarding theoptimization of compositecylindricalshells, Huand Wang (1992) studied these structures with and without cutouts, and $\mathrm{Hu}$ and Tsai (1999) analyzed the fundamental frequencies. Tabu-embedded simulated annealing was applied for the optimal stacking sequence designed by Rao and Arvind (2007). Stacking sequence optimization of laminated cylindrical shells for buckling and free vibration using genetic algorithm and neural networks have been investigated by Gharib and Shakeri
(2010). Mota Soares et al. (1995) researched the sensitivity analysis of the optimization of general thin shell structures made of composite materials. Chern and Chao (2000) studied the natural frequencies of laminates in spherical, cylindrical, planed, and curved panel geometries by a three-dimensional theory. They also presented the comparison of natural frequency via a three-dimensional augmented energy variation approach.

The Layerwise optimization was applied for maximizing the fundamental frequency of laminated panels by Narita and Robinson (2006). Goupee and Vel (2006) proposed a methodology to optimize the natural frequencies of functionally grade structures. Rao and Lakshmi (2009) suggested an algorithm for solving multi-objective optimization problem. Hybrid scatter search algorithm was employed to solve stacking sequence optimization of hybrid fiber reinforced composite plate, cylindrical shell, and pressure vessels. A comparison of different shell theories considering nonlinear vibrations of laminated circular cylindrical shells was obtained by Amabili (2011). Therefore, this work addressed the development of an ACO algorithm applied to the layup design of composite cylindrical shells. Numerical tests were performed for the layup optimization of laminated cylindrical shells with holes aiming at maximizing the fundamental frequency.

\section{ANT COLONY OPTIMIZATION APPLIED TO LAMINATED CYLINDRICAL SHELLS}

ACO wasinspired from the studies of real ant colonies foraging behavior. Pheromone is a chemical product that ants deposit on the ground. Its concentration influences probabilistically the choice of best way. This kind of behavior is called stigmergy and is the mechanism that controls communication among the ants, and make them take the shortest paths. Pheromone matrix is the basic information processed by ACO to find the solutions following the way with more concentration of this substance.

Artificial ants are used to construct solutions for laminated composite cylinders by choosing probabilistically the orientation of the laminate stacks. An artificial ant means a colony of artificial ones that search the best ways. Figure 2 presents the procedure of ConstructionAntsSolutions. The solutions are built on the past search experience based on the level of pheromone and the heuristic matrix, which brings information about the problem to be solved. The heuristic information is the parameter related to problem, for this case the relation in function of total weight of laminated was considered. The ACO procedure starts with a random 
selection for the layers' orientations. After the artificial ants have finished building the first laminated configurations, the pheromone is released with evaporation and the new amount of pheromone is deposited, based on the best solutions found at local and global iterations. The $\operatorname{arc}(\mathrm{i}, \mathrm{j})$ denotes the stacking sequence, qualifying the pheromone trail $\tau_{i j}$ for local updating rule, in function of frequency value.

The algorithm stops when the maximum number of iterations or objective function evaluations is reached. It is assumed that the laminate is symmetric and balanced with the allowable ply orientations $(0 / 0 /+45 /-45 / 90 / 90)$. Also, to prevent matrix cracking, a maximum number of four contiguous plies with the same orientation is imposed as a constraint (Le Riche and Haftka, 1993).

\section{NUMERICAL RESULTS}

The case studies considered herein are the maximization of fundamental frequency of composite cylindrical shells. ACO was implemented in Matlab platform and was linked to finite element (FE) code Abaqus in order to compute the frequency response. The ply angles orientation considered for the layers are $0^{\circ}, \pm 45^{\circ}$, and $90^{\circ}$. The laminates are considered symmetric and balanced. The first step of simulation is done by ACO, which defines the ply angles and save them in a file that was read in script wrote in Python. This script is connected to Abaqus to send the stacking sequence and receive the computed results. The results are sent again to ACO to compute the optimization. Finite element solution is processed for the fundamental frequency, and the correspondent value is returned to ACO and so on, for searching the maximal fundamental frequency.

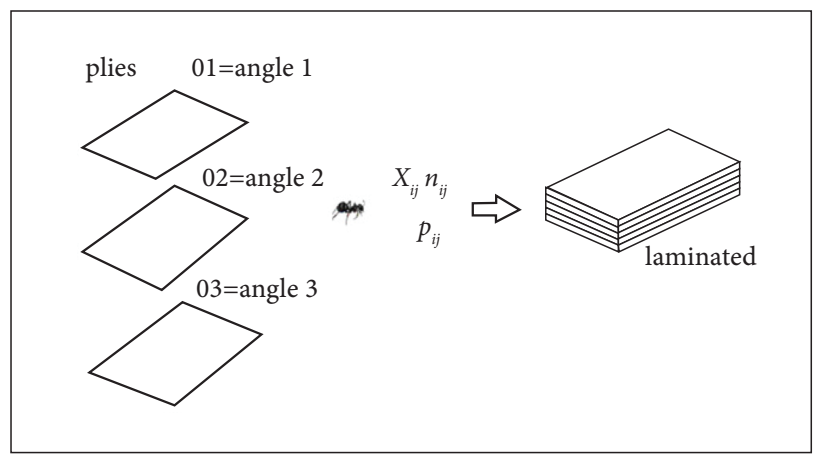

Figure 2. ConstructionAntsSolutions procedure.
In order to validate the finite element code solution, the first numerical studies were a frequency calculation obtained by finite elements, compared to the results obtained by Poore et al. (2008). The problem solved was a cylindrical shell with radius $(R=0.05)$ inch, length $(L=2 R)$, thickness $(h=0.02 R)$. The material property values were: $E_{22}=1.0 \times 10^{6}$ psi, $E_{11}=25 E_{22}, G_{12}=G_{13}=0.5 \mathrm{E}_{22}, v_{12}=0.25$, and $\rho=1.0$. Simply supported (SS3) boundary conditions were adopted along the edges of the shell.

Three different stacking sequences were simulated. Table 1 presents the comparison of the normalized fundamental frequency, which is defined as Eq. 4:

$\bar{\omega}=\omega\left(R^{2} / h\right) \sqrt{\left(p / E_{22}\right)}$

where, $\omega$ is the fundamental frequency.

\section{OPTIMIZATION PROBLEM}

The optimization problem is formulated as Eq. 5: Find: $\theta^{\mathrm{k}}, \theta^{\mathrm{k}} \in\left\{0_{2}, \pm 45,90_{2}\right\}, k=1$ to $n$

Maximize: Fundamental frequency

Subject to: - Symmetric and balanced laminate

- Maximum number of contiguous plies with the same orientation $=4$

where,

$\theta^{\mathrm{k}}$ is the orientation of each stack of the laminate, and $n$ is the total number of stacks.

Each stack is composed of two layers with the same orientation, but opposite signs (for instance, $\pm 45^{\circ}$ ) to guarantee balance. Still, considering the symmetry of the laminate, $n$ corresponds to the total number of layers $N_{\mathrm{L}}$ divided by four $\left(n=N_{\mathrm{L}} / 4\right)$. Graphite/epoxy material was used. Their properties are, $E_{1}=128 \mathrm{GPa}, E_{2}=11 \mathrm{GPa}, G_{12}=G_{13}=4.48 \mathrm{GPa}, v_{12}=0.25$, and $p=1500 \mathrm{~kg} / \mathrm{m}^{3}$. Figure 3 shows the laminated cylindrical shell.

The dimensions of the cylindrical shell structure are: radius $(R=0.1 \mathrm{~m})$, length $(L=0.2 \mathrm{~m})$, and ply thickness ( $h=0.125 \mathrm{~mm}$ ) Simply supported edges (SS3) as boundary conditions were adopted for the simulations.

Table 1. Normalized fundamental frequency comparison.

\begin{tabular}{|c|c|c|}
\hline Layup & $\bar{\omega}$ (Poore et al., 2008) & $\bar{\omega}$ (FE code) \\
\hline$\left[90^{\circ} / 0^{\circ}\right]_{2}$ & 19.473 & 19.582 \\
\hline$\left[ \pm 45^{\circ}\right]_{S}$ & 28.206 & 29.000 \\
\hline$\left[ \pm 60^{\circ}\right]_{S}$ & 25.909 & 26.515 \\
\hline
\end{tabular}




\section{CYLINDRICAL SHELL WITHOUT CUTOUT}

Firstly, the fundamental frequency was obtained considering all the 64 plies oriented with the same angle $\left(0^{\circ}, \pm 45^{\circ}\right.$ or $\left.90^{\circ}\right)$. The results are showed in Table 2 and the corresponding cylindrical geometry with the finite element mesh is presented in Fig. 4.

In the finite element analysis, the laminated cylindrical shells are modeled by eight-node thick shell elements with six degrees of freedom per node using reduced integration.

The optimization was carried out on a single processor computer with $3.0 \mathrm{GHz}$ Intel Core $2 \mathrm{CPU}$ including 4GB memory, and the total computational time needed was about 75 hours (150 evaluations, per hour). The optimized stacking sequence $\left(\theta_{k}\right)$, the corresponding fundamental frequency $\left(f_{n}\right)$, and the number of objective function evaluations (N) to reach the solution are presented in Table 3 .

Table 4 presents the comparison of the optimized frequency against the ones obtained by all plies with the same orientation $\left(0^{\circ}, \pm 45^{\circ}\right.$ or $\left.90^{\circ}\right)$. Despite the small gain of the optimized laminate compared to the one with all plies oriented at $\pm 45^{\circ}$, the percentage gains against the $0^{\circ}$ and $90^{\circ}$ laminates were high, achieving 95.71 and $71.23 \%$, respectively.

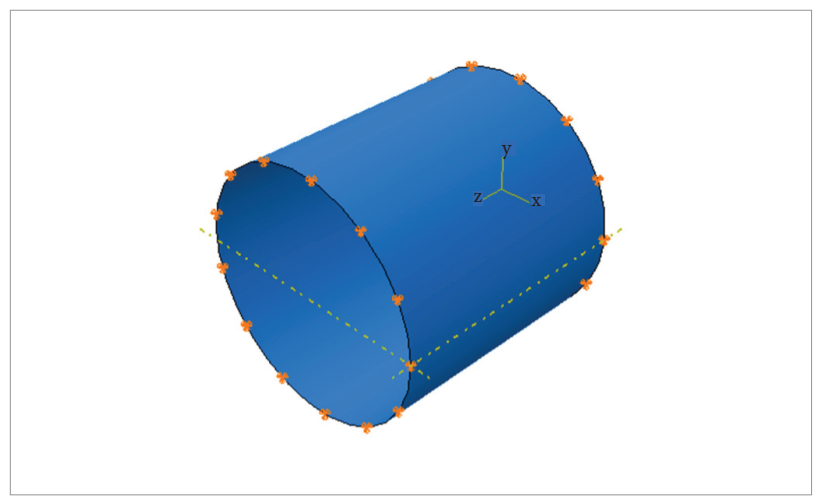

Figure 3. Laminated cylindrical shell.

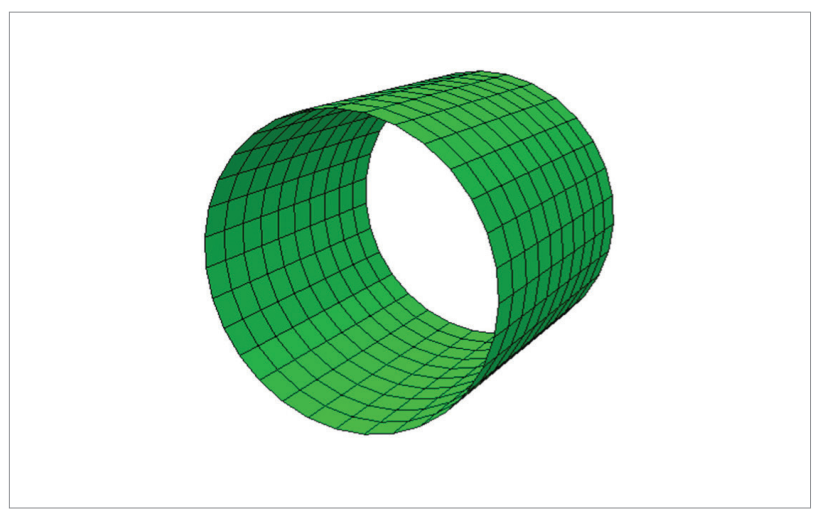

Figure 4. Finite element mesh of the cylindrical shell without cutout.
Figure 5 presents the fundamental vibration modes of the cylindrical shells with the cutout for different stacking sequences. It can be noted that the modes shown are very similar to each other, except those for the stacking sequence $\left[90^{\circ}{ }_{32}\right]_{S}$.

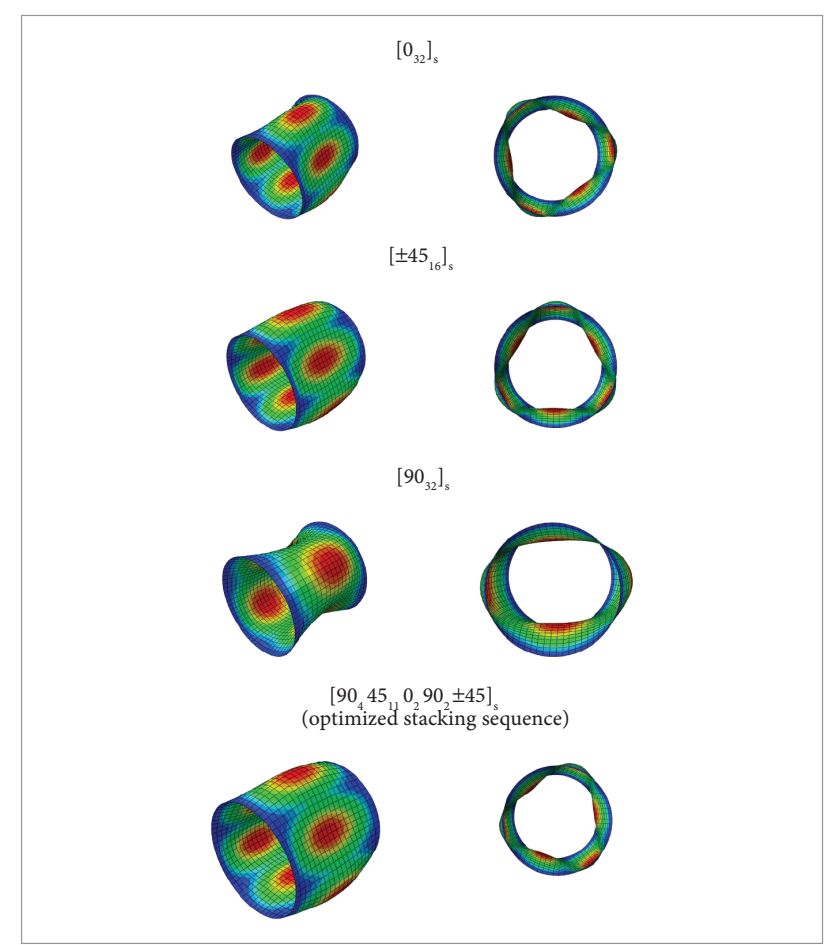

Figure 5. Fundamental vibration mode for different stacking sequences (structure with the cutout).

Table 2. Fundamental frequency for the structure without cutout.

\begin{tabular}{|c|c|}
\hline Layup & Frequency (Hz) \\
\hline$\left[0_{32}\right]_{S}$ & 1803.40 \\
\hline$\left[ \pm 45_{16}\right]_{S}$ & 3450.30 \\
\hline$\left[90_{32}\right]_{s}$ & 2061.20 \\
\hline
\end{tabular}

Table 3. Optimized results for the structure without cutout.
$f_{n}$
$3529.40(\mathrm{~Hz})$
$\theta^{k}$
$\left[\begin{array}{llll}90_{4} & 45_{11} & 0_{2} & 90_{2} \pm 45\end{array}\right]_{S}$
$\mathrm{n}$
11250

Table 4. Comparison of frequencies (structure without cutout].

\begin{tabular}{|c|c|c|}
\hline Layup & Frequency (Hz) & Difference [\%] \\
\hline$\left[0_{32}\right]_{S}$ & 1803.40 & 95.71 \\
\hline$\left[ \pm 45_{16}\right]_{S}$ & 3450.30 & 2.29 \\
\hline$\left[90_{32}\right]_{S}$ & 2061.20 & 71.23 \\
\hline
\end{tabular}




\section{CYLINDRICAL SHELL WITH A CUTOUT}

In this case, the cylindrical shell geometry is more complex than the precedent one. Figure 6 shows the cutout geometry, and Fig. 7 demonstrates the cylindrical shell with the cutout and the corresponding finite element mesh. Because we are interested in the vibration frequencies and not in obtaining the stress field or the maximum stress, no mesh refinement was done in the cutout region.

Firstly, the fundamental frequency was obtained considering all the 64 plies oriented with the same angle $\left(0^{\circ}\right.$, $\pm 45^{\circ}$ or $90^{\circ}$ ). These results are showed in Table 5 .

The optimized results for the structure with the cutout are showed in Table 6. Table 7 presents the comparison of the optimized frequency against the ones obtained by all plies with the same orientation $\left(0^{\circ}, \pm 45^{\circ}\right.$ or $\left.90^{\circ}\right)$. Again, as in the previous case, despite the small gain of the optimized laminate compared to the one with all plies oriented at $\pm 45^{\circ}$, the percentage increases against the $0^{\circ}$ and $90^{\circ}$ laminates with 90.67 and $69.69 \%$, respectively.

As the total number of evaluations used for this case was the same as the precedent one $(\mathrm{n}=10512)$ and the time for each finite element evaluation was also approximately the same, the total computational time remained similar to the previous case (about 70 h).

Figure 8 presents the fundamental vibration modes of the cylindrical shells with the cutout for different stacking sequences. It can be noted that the modes are very similar to each other.

Table 5. Fundamental frequency for the structure with the cutout.

\begin{tabular}{|c|c|}
\hline Layup & Frequency (Hz) \\
\hline$\left[0_{32}\right]_{S}$ & 1777.20 \\
\hline$\left[ \pm 45_{16}\right]_{S}$ & 3311.90 \\
\hline$\left[90_{32}\right]_{S}$ & 1996.90 \\
\hline
\end{tabular}

Table 6. Optimized results for the structure with the cutout.
$f_{n}$
$\theta^{k}$
$\mathrm{n}$
$3388.60(\mathrm{~Hz})$

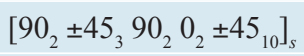
10512

Table 7. Comparison of frequencies (structure with the cutout).

\begin{tabular}{|c|c|c|}
\hline Layup & Frequency $(\mathrm{Hz})$ & $\%$ \\
\hline$\left[0_{32}\right]_{S}$ & 1777.20 & 90.67 \\
\hline$\left[ \pm 45_{16}\right]_{S}$ & 3311.90 & 2.32 \\
\hline$\left[90_{32}\right]_{S}$ & 1996.90 & 69.69 \\
\hline
\end{tabular}

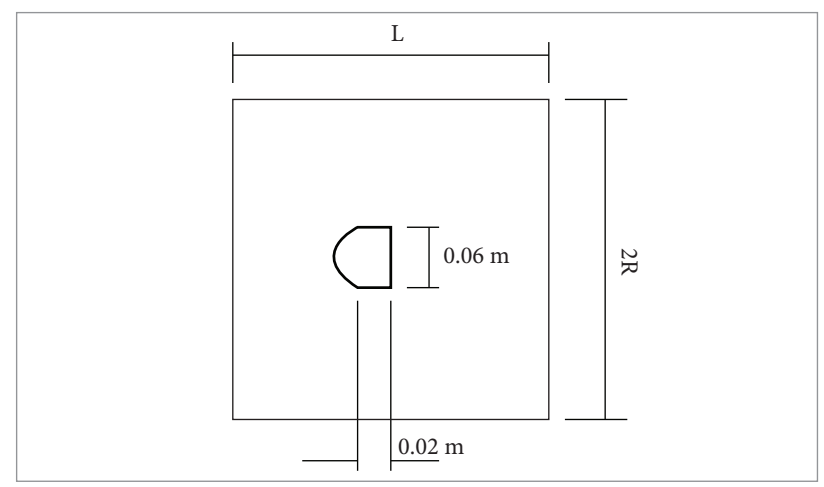

Figure 6. Cutout geometry.

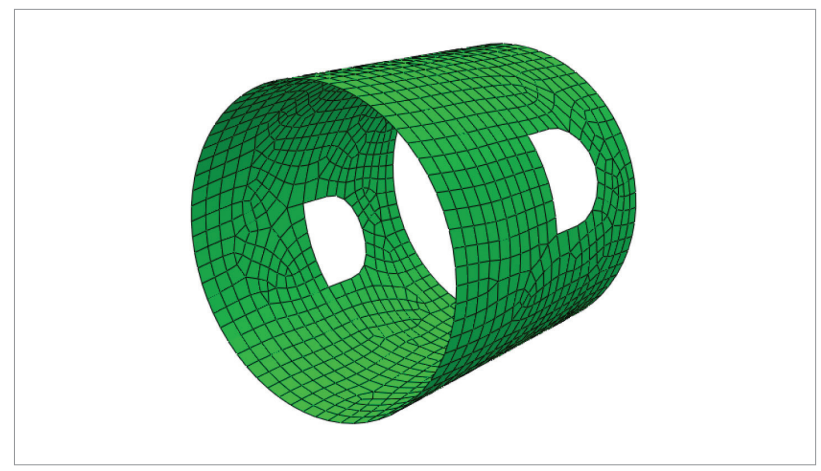

Figure 7. Finite element mesh of the cylindrical shell with cutout.

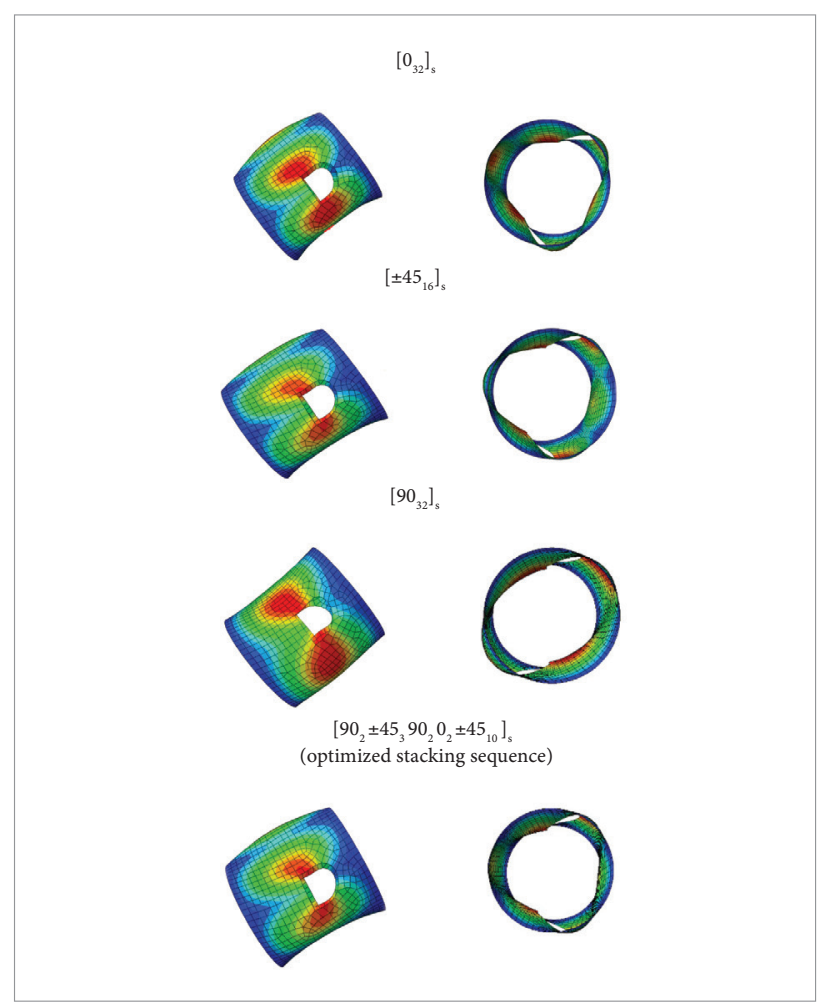

Figure 8. Fundamental vibration modes for different stacking sequences (structure with the cutout). 


\section{CONCLUSIONS}

Cylindrical shells tailored with reinforced composite material were optimized. The fundamental frequency maximization was performed by an ACO algorithm, coupled with a finite element code to obtain the structural response. The ply angle orientations, i.e., stacking sequences, were obtained for structures without and with a cutout. The numerical results were presented and discussed in both cases.
We concluded that the ant colony metaheuristic can be efficiently applied to find optimal stacking sequences of cylindrical shells with cutout or complex geometries. As a further investigation, we can apply this method to multiobjective optimization, for example, maximizing the fundamental frequency and the buckling load. Another improvement of this approach would be to include the material as a design variable.

\section{REFERENCES}

Amabili, M., 2011, "Nonlinear vibrations of laminated circular cylindrical shells: Comparison of different shell theories", Composite Structures, Vol. 94, pp. 207-220.

Barbero, E. J., Reddy, JN. andTeply, J.L., 1990, "Generaltwo-dimensional theory of laminated cylindrical shells", AIAA Journal, Vol. 28, No. 3, pp. 544-553.

Chern, Y. and Chao, C.C., 2000, "Comparison of natural frequencies of laminates by 3-D theory, Part II: curved panels", Journal of Sound and Vibrations, Vol. 230, pp. 1009-1030.

Dorigo, M. and Stützle, T., 2004, "Ant colony optimization", MIT, Cambridge, USA.

Ganapathi, M. and Haboussi, M., 2003, "Free vibrations of thick laminated anisotropic non-circular cylindrical shells", Composite Structures, Vol. 60, pp. 125-133.

Ganapathi, M., Patel, B.P. and Patel, H.G., 2004, "Free flexural vibration behavior of laminated angle-ply elliptical cylindrical shells", Computers and Structures, Vol. 82, pp. 509-518.

Gharib, A. and Shakeri, M., 2010, "Stacking sequence optimization of laminated cylindrical shells for buckling and free vibration using genetic algorithm and neural networks", Second International Conference on Engineering Optimization, Lisbon.

Goupee, A. and Vel, S.S., 2006, "Optimization of natural frequencies of bidirectional functionally graded beams", Structural Multidisciplinary Optimization, Vol. 32, pp. 473-484

Heyliger, P.R. and Jilani, A., 1993, "Free vibrations of laminated anisotropic cylindrical shells", Journal of Engineering Mechanics, Vol. 119, pp. 1062-1077.

$\mathrm{Hu}, \mathrm{H}$. and Tsai, J., 1999, "Maximization of the fundamental frequencies of laminated cylindrical shells with respect to fiber orientations", Journal of Sound and Vibration, Vol. 225, No. 4, pp. 723-740.

$\mathrm{Hu}, \mathrm{H}$. and Wang, S., 1992, "Optimization for buckling resistance of fiber-composite laminate shells with and without cutouts", Composite Structures, Vol. 22, pp. 03-13.

Irisarri, F.X., Laurin, F., Leroy, F.H. and Maire, J.F., 2011, "Computational strategy for multiobjective optimization of composite stiffened panels", Composite Structures, Vol. 93, pp. 1158-1167.
Lam, K.Y. and Qian, W., 2000, "Free vibration of symmetric angleply thick laminated composite cylindrical shells", Composites Part B, Vol. 31, pp. 345-354.

Le Riche, R. and Haftka, R., 1993, "Optimization of Laminate Stacking Sequence for Buckling Load Maximization by Genetic Algorithm", AIAA Journal, Vol. 31, pp. 951-956.

Mota Soares, C.M., Franco Correia, V., Mateus, H. and Herskovits, J., 1995, "A discrete model for the optimal design of thin composite plateshell type structures using a two-level approach", Composite Structures, Vol. 30, pp. 147-157.

Narita, Y. and Robinson, P., 2006, "Maximizing the fundamental frequency of laminated cylindrical panels using layerwise optimizations", International Journal of Mechanical Sciences, Vol. 48, pp. 1516-1524.

Poore, A.L., Barut, A.E. and Madenci, E., 2008, "Free vibration of laminated cylindrical shells with a circular cutout", Journal of Sound and Vibration, Vol. 312, pp. 55-73.

Rao, A. and Arvind, N., 2007, "Optimal stacking sequence design of laminate composite structures using tabu embedded simulated annealing", Structural Engineering and Mechanics, Vol. 25, pp. 239-268.

Rao, A.R.M. and Lakshmi, K., 2009, "Multi-objective optimal design of hybrid laminate composite structure using scatter search", Journal of Composite Materials, Vol. 43, pp. 2157-2182.

Shafei, E. and Kabir, M.Z., 2011, "Dynamic stability optimization of laminated composite plates under combined boundary loading", Applied Composite Material, Vol. 18, pp. 539-557.

Sharma, A.K. and Mittal, N.D., 2010, "Review on stress and vibration analysis of composite plates", Journal of Applied Sciences, Vol. 10, No. 23, pp. 3156-3166.

Soldatos, K.P., 1994, "Review of three dimensional dynamic analyses of circular cylinders and cylindrical shells", American Society of Mechanical Engineers, Vol. 47, No. 10, pp. 501-516.

Todoroki, A., Shinoda, T., Mizutani, Y. and Matsuzaki, R., 2001, "New surrogate model to predict fracture of laminated CFRP for structural optimization", Journal of Computational Science and Technology, Vol. 5, No. 1, pp. 26-37. 